\title{
Immunosuppressive therapy in idiopathic multifocal fibrosis. A case report
}

\author{
Damian Franzen ${ }^{1}$, Hans-Georg Brochhagen ${ }^{2}$, Efim Benenson ${ }^{3}$ \\ ${ }^{1}$ Praxis für Herz und Lungenkrankheiten, Cologne, Germany; \\ ${ }^{2}$ Radiologische Praxis, Cologne, Germany; \\ ${ }^{3}$ Praxis für Rheumatologie, Cologne, Germany. \\ Email: info@damianfranzen.de
}

Received 15 May 2011; revised 20 June 2011; accepted 4 July 2011.

\begin{abstract}
Multifocal fibrosis is a rare condition occurring at any age and characterized by chronic inflammation, soft tissue proliferation and subsequent fibrosis of various vascular connective tissues. Following diagnosis in a 43-year accountant with multiple vascular lesions, the patients was treated successfully with azathioprine and steroids over a period of 3 years. Fatigue and sweating, serologic sign of inflammation and radiological imaging studies normalized completely. Twenty four months after termination of therapy, there were no clinical or serologic signs of relapse.
\end{abstract}

Keywords: Periaortitis; Multifocal Fibrosis; Retroperitoneal Fibrosis

\section{INTRODUCTION}

Retroperitoneal fibrosis (Morbus Ormond) is a rare disease characterized by chronic inflammation, soft tissue proliferation and subsequent fibrosis of the retroperitoneum typically involving and obstructing the ureters [1]. If fibrous lesions occur at different sites of the body, the condition is termed multifocal fibrosis [2,3]. The etiology of these conditions is unknown, however new data support a close linkage to IgG-4 expressing plasma cells (Hyper-Ig4 disease) [4,5]. Retroperitoneal fibrosis may be idiopathic or secondary to malignancy, a specific drug history, radiation therapy or autoimmune disease. Due to the compressive effects of retroperitoneal fibrosis treatment has been a domain of surgery. Basing on pathophysiological considerations several authors however support a non-surgical approach but reported treatment regimens with systemic steroids remain anecdotal [1,6-8].

In the following we report on a long-term successful immunosuppressive therapy in a male patient with idiopathic multifocal fibrosis.

\section{CASE REPORT}

In 2006, a 43-year old accountant was referred for clarification of a persistent elevated sedimentation rate following a dental procedure. He had been complaining of fatigue, sweating and exertional shortness of breath which had been initially addressed to his gross overweight (136 kg, $194 \mathrm{~cm}$, body mass Index 36). His past medical and the family history were unremarkable. He was nonsmoker, married, father of 2 kids and living in the suburbs of a greater German city.

The physical exam was unremarkable, the blood pressure 131/78 mmHg. No lymph nodes or an enlarged spleen were palpable. The initial laboratory examinations revealed a sedimentation rate of $80 \mathrm{~mm} / \mathrm{h}$, a C-reactive protein of $73 \mathrm{mg} / \mathrm{dl}$ (normal range up to $5 \mathrm{mg} / \mathrm{dl}$ ) and slight anemia with a hemoglobin count of $13.0 \mathrm{~g} / \mathrm{dl}$ (normal range 14 - $18 \mathrm{~g} / \mathrm{dl}$ ). Serum-fibrinogen was elevated (415 mg/dl, normal range 180 - $350 \mathrm{mg} / \mathrm{dl}$ ) and immunoglobulin G slightly diminished (6.9 g/dl, normal range 7 - $16 \mathrm{~g} / \mathrm{dl}$ ). Liver enzymes, electrolytes and creatinine were normal, serial blood cultures sterile.

A CT scan of the thorax revealed a hypo dense mass adjacent to the aortic arch slightly accumulating contrast medium (Figure 1). An oral and intravenous contrast enhanced computer tomography of the abdomen revealed hypo dense masses around the aorta and the iliac arteries (Figure 2). T1 and T2-weighted magnetic resonance imaging (MR) confirmed the presence of oval shaped masses of intermediate soft-tissue intensity adjacent to the lateral aortic arch and surrounding the abdominal aorta and iliac arteries. MR of the heart and the thoracic vessel including the thoracic and abdominal aorta showed no abnormalities, plaque or aneurysm.

Following diagnostic exclusion of a malignancy, an autoimmune disease and/or a specific drug history the diagnosis of idiopathic multifocal fibrosis was made. Due to the close neighborhood to great vessel no attempt was 


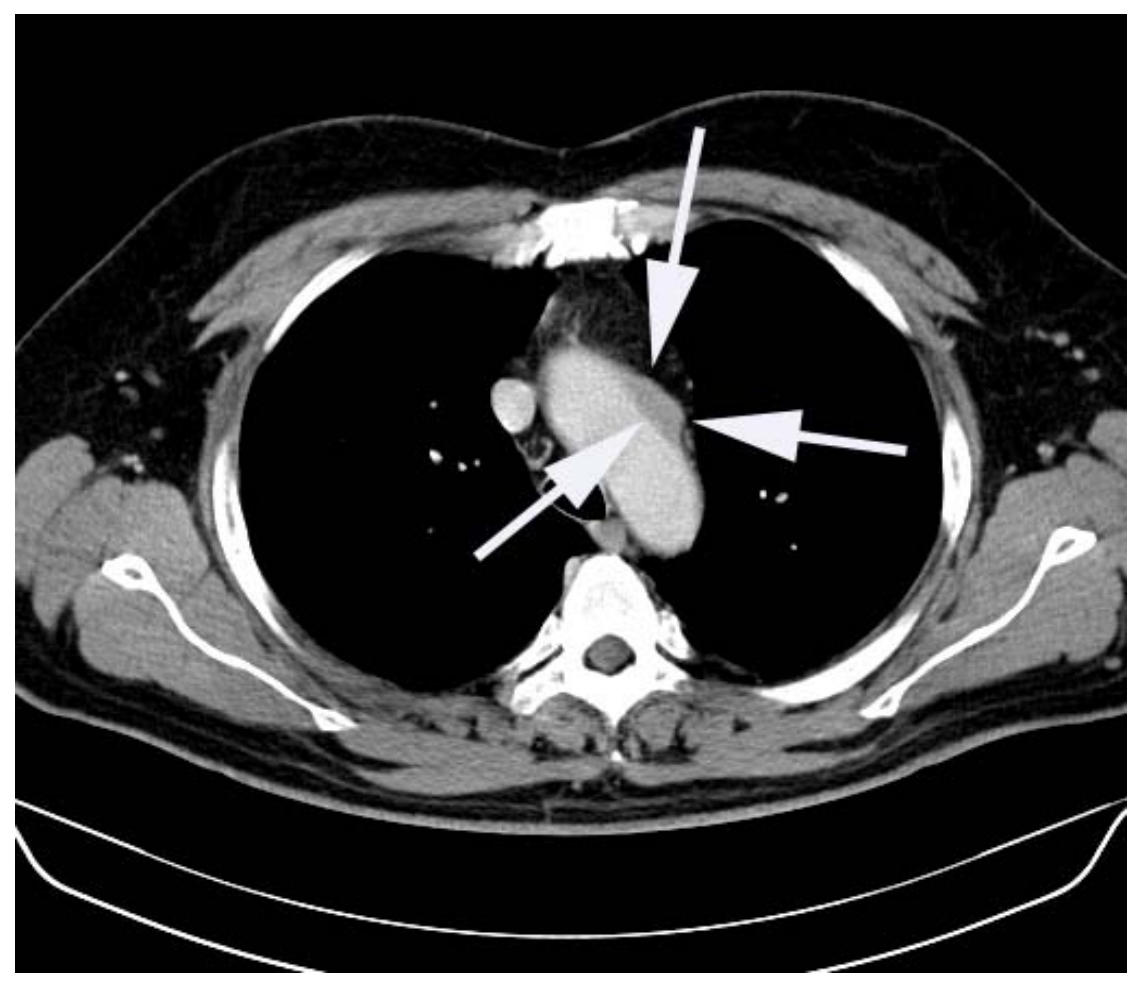

Figure 1. Computed tomography of the thorax. Arrows point at soft tissue proliferation around the aortic arch.

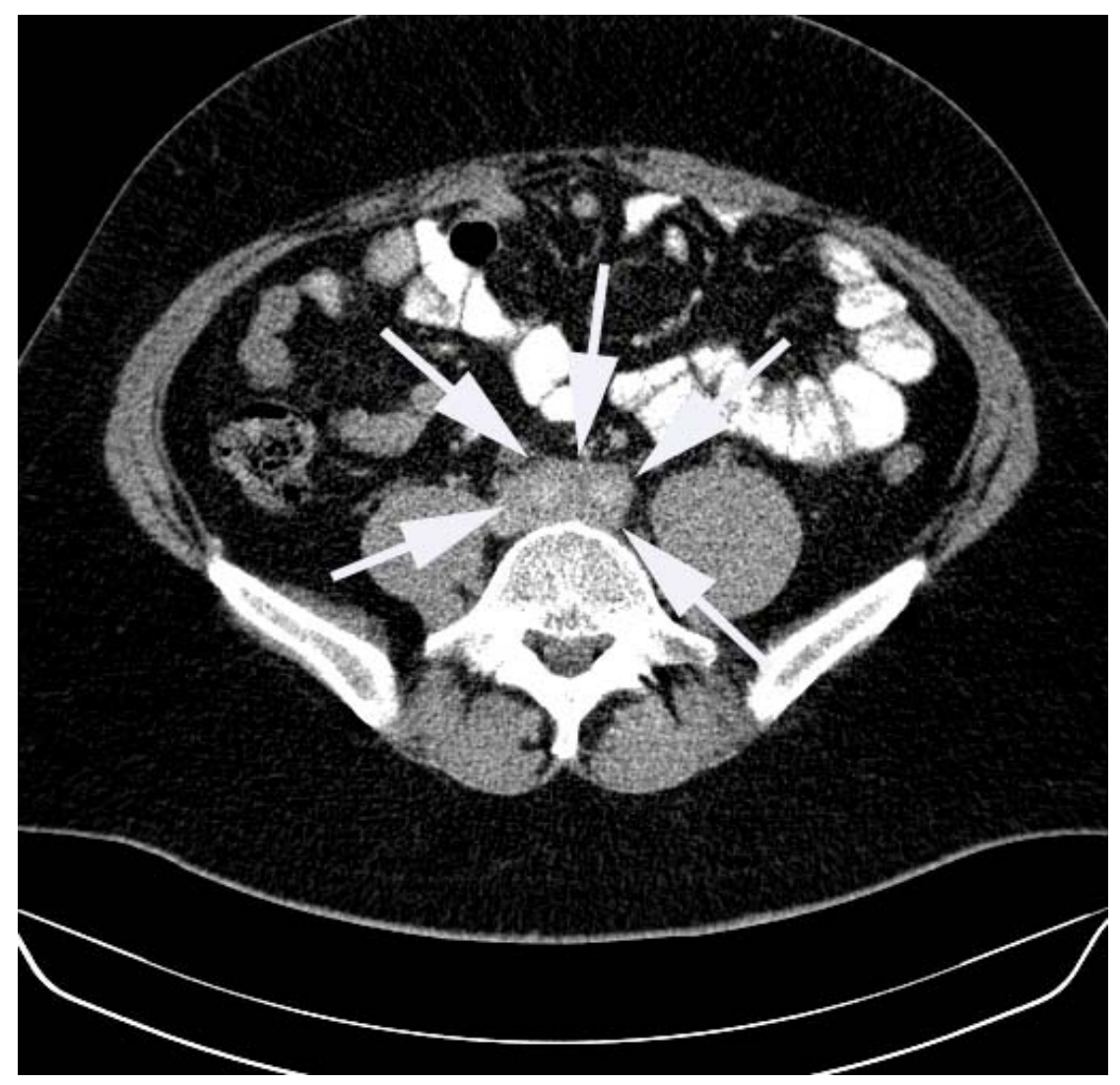

Figure 2. Computed tomography of the abdomen. Arrows point at soft tissue proliferation. 
made to perform a tissue biopsy. At the discretion of the rheumatologist a therapy with $20 \mathrm{mg}$ prednisolone/day was instituted leading to normalization of the sedimentation rate and the C-reactive protein within 2 weeks. Considering a long term treatment, azathioprine $125 \mathrm{mg} /$ day was instituted and prednisolone tapered down to 10 $\mathrm{mg}$ /day within the following two weeks. Because of bilateral femoral head necrosis and development of a symptomatic diabetes mellitus several months following treatment start, attempts to reduce the dose of steroids were started however failed as demonstrated by rapid increases of the sedimentation rate and the C-reactive protein. Following 3 years of the azathioprine and oral steroid therapy, treatment was discontinued in 2009. All radiographic findings were resolved (Figures 3 and 4), the sedimentation rate and the C-reactive protein were normalized. At follow up 24 months after termination of therapy, the patient was still free of any symptoms or serologic signs of relapse. The CT scan of the thorax revealed no infiltration of the aorta or connective tissues.

\section{DISCUSSION}

Idiopathic multifocal fibrosis is a rare condition occ- urring at any age and characterized by chronic inflammation, soft tissue proliferation and subsequent fibrosis of various vascular connective tissues. It should be differentiated from inflammatory vascular diseases, which affect the vascular wall primarily and then extend to the connective tissues [9]. Due to the heterogeneity of vascular involvement and clinical presentation these diseases are referred to the large entity of chronic periaortitis [10]. Apart from the classical retroperitoneal fibrosis (M. Ormond), fibrosis may involve all other abdominal organs, the mediastinum, the orbita, different glands, and many other organs $[1-3,5,6]$. The diagnosis is based on radiographic findings, biopsy and exclusion of secondary forms. Although the syndrome appears autoimmune in nature, to date there are no specific immunologic markers $[11,12]$.

Whereas obstruction of the ureters in retroperitoneal fibrosis favors a surgical approach, anti-inflammatory medical regimens have been instituted to stop connective tissue proliferation and systemic fibrosis [9]. Kardar and coworkers treated 11 patients with idiopathic retroperitoneal fibrosis with oral prednisolone over a period of 2 years and reported good clinical response and regression.

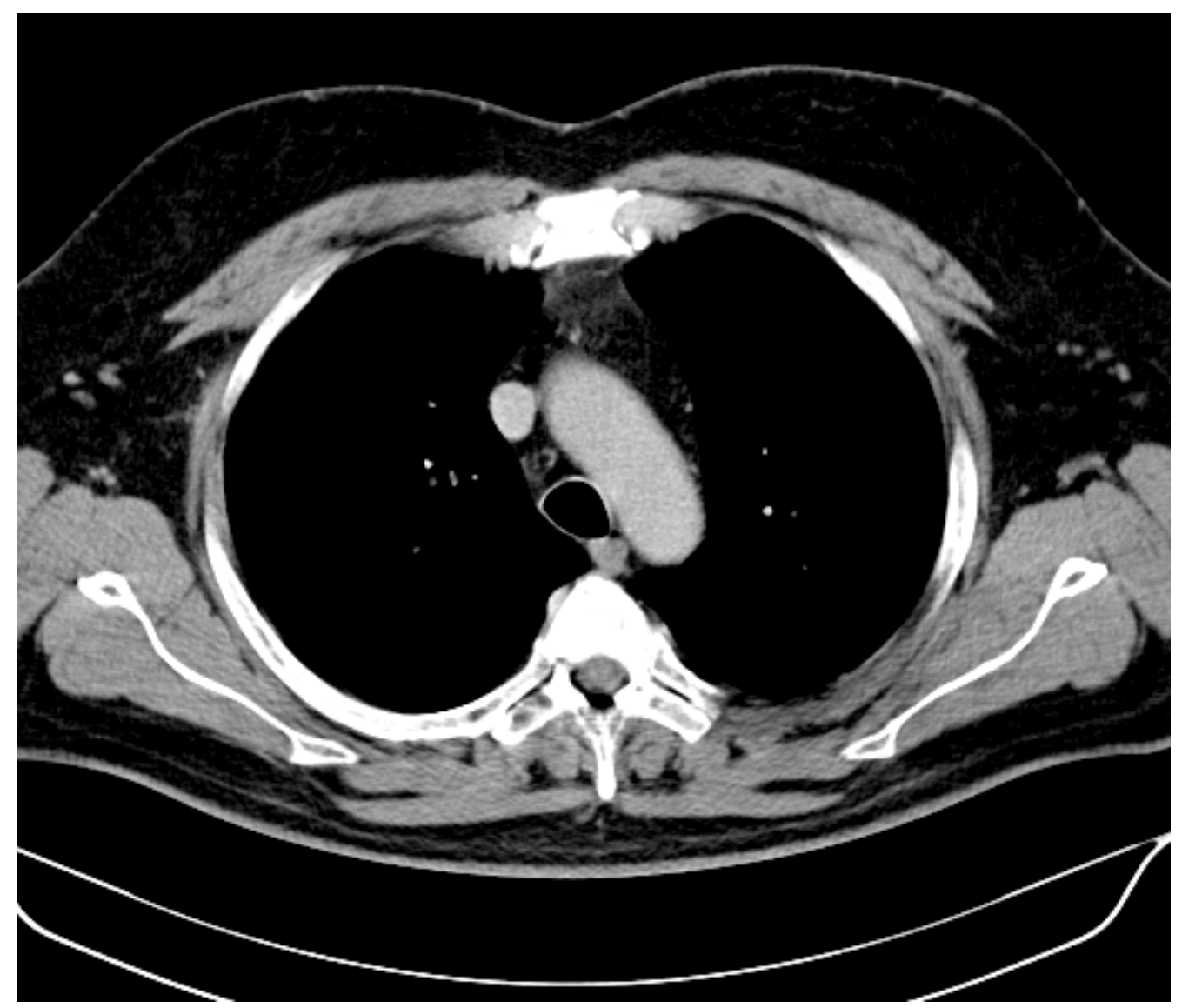

Figure 3. Computed tomography of the thorax following longterm treatment with prednisolone and azathioprine demonstrating resolution of connective tissue proliferation. 


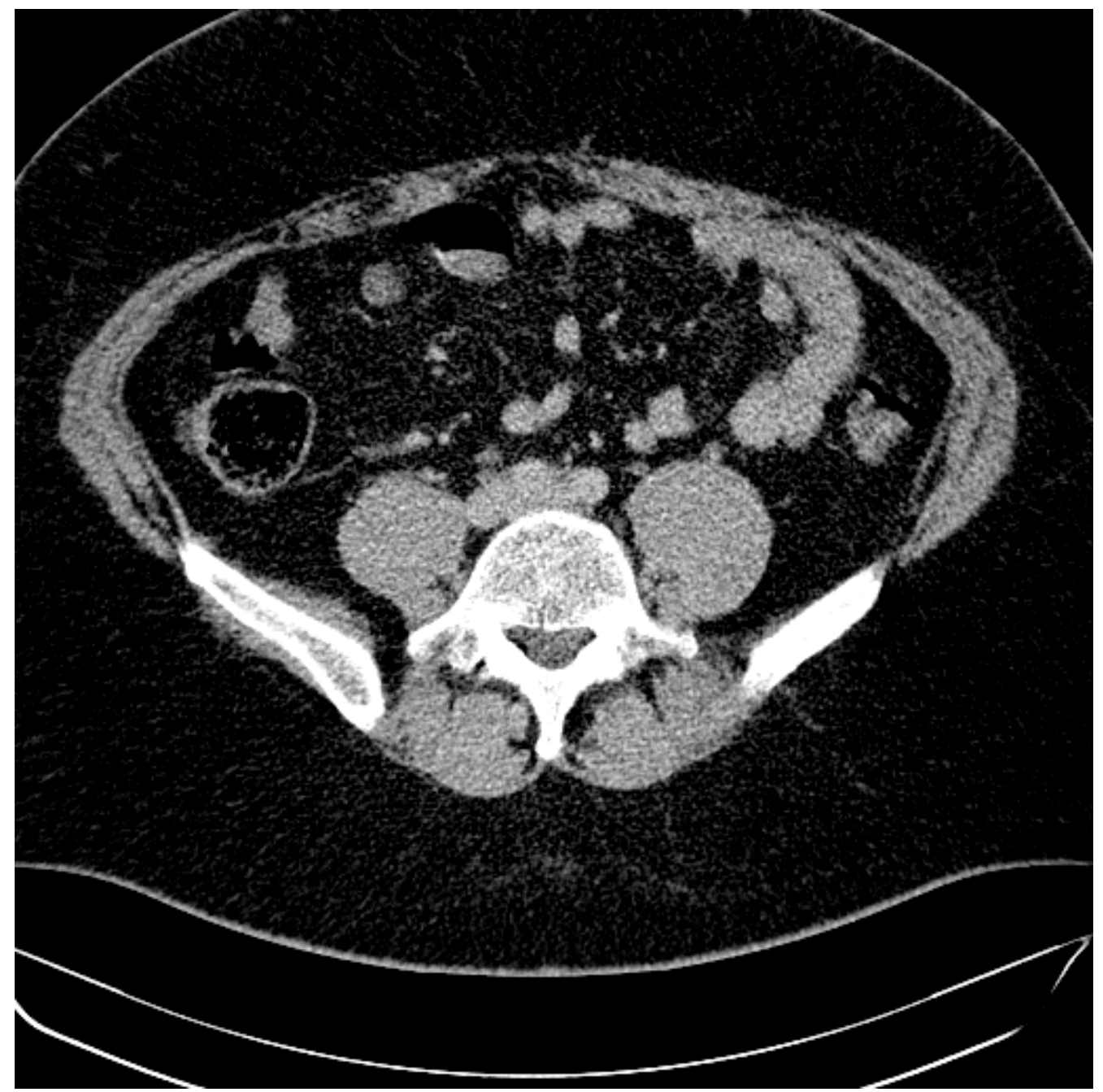

Figure 4. Computed tomography of the abdomen following longterm treatment with prednisolone and azathioprine demonstrating resolution of connective tissue proliferation

in $9(82 \%)$ patients [8]. Although larger studies are missing, steroid administration alone or in conjunction with surgery are currently thought to be the best way of treatment for retroperitoneal fibrosis $[1,8]$. As stated by many others, immunosuppressive therapy treatment should extend to several years due to the relapsing course of the fibrosing syndrome [1,12]. Apart from systemic steroid several others immunosuppressant therapies such as with cyclophosphamide, azathioprine, and mycophenolate mofetil have been employed successfully in retroperitoneal fibrosis $[1,13]$.

Although a common pathologic mechanism suggested, multifocal fibrosis may differ from retroperitoneal fibrosis. The multiorgan involvement in multifocal fibrosis obligetory requires a systemic treatment approach. In the presented case clinical and radiographic findings were compatible with the diagnosis of idiopathic multifocal fibrosis. The reported responses to medical therapy are variable.
Clinical endpoints are general symptoms, sedimentation rate, and imaging studies with abdominal computed tomography and magnetic resonance imaging. We employed a low dose concomitant corticosteroid and azathoprine treatment over a period of 3 years which yielded a normalization of clinical and serologic markers as well as complete radiographic resolution of connective tissue proliferation. Thus, it seems that tissue proliferation may be reversible and the term fibrosis misleading [14].

\section{REFERENCES}

[1] van Bommel, E.F.H. (2002) Retroperitoneal fibrosis. The Netherlands Journal of Medicine, 60, 231-242.

[2] Flückiger, B., Bunne, W., Kuhn, M. and Reinhart, W.H. (2006) Idiopathische multifokale Fibrose. Schweizerisches Medizinisches Forum, 6, 83-84.

[3] Szarf, G. and Bluemke, D.A. (2005) Case 83: Multifocal fibrosclerosis with mediastinal-retroperitoneal involve- 
ment, Radiology, 235, 829-832. doi:10.1148/radiol.2352030088

[4] Neild, G.H., Rodriguez-Justo, M., Wall, C. and Connolly, J.O. (2006) Hyper-IgG4 disease: Report and characterisation of a new disease. BMC Medicine, 4, 1-25. doi:10.1186/1741-7015-4-23

[5] Kamisawa, T. and Okamoto, A. (2008) IgG-4 related sclerosing disease. World Journal of Gastroenterology, 14, 3948-3955. doi:10.3748/wjg.14.3948

[6] Maturen, K.E., Sundaram, B., Marder, W. and Swartz, R.D. (2011) Coronary artery involvement in idiopathic retroperitoneal fibrosis: Computed tomographic findings. Journal of Thoracic Imaging, (Epub ahead of print).

[7] Novak, I., Moravek, P., Base, J. and Svab, J. (1994) Longterm results in patients with retroperitoneal fibrosis. Rozhledy v Chirurgii, 73, 287-290.

[8] Kardar, A.H., Kattan, S., Lindstedt, E. and Hanash, K. (2002) Steroid therapy for idiopathic retroperitoneal fibrosis: Dose and duration. Journal of Urology, 168, 550555. doi:10.1016/S0022-5347(05)64677-0

[9] Warnatz, K., Keskin, A.G., Uhl, M., Scholz, C., Katzenwadel, A., Vaith, P., Peter, H.H. and Walker, U.A. (2005)
Immunosuppressive treatment of chronic periaortitis: A retrospective study of 20 patients with chronic periaortitis and a review of the literature. Annals of the Rheumatic Diseases, 64, 828-833. doi:10.1136/ard.2004.029793

[10] Vaglio, A. (2006) Retroperitoneal fibrosis. The Lancet, 367, 241-251.

[11] Vaglio, A., Greco, P., Conradi, D., Palmisano, A., Martorana, D., Ronda, N. and Buzio, C. (2006) Autoimmune aspects of chronic periarotitis. Autoimmunity Reviews, 5, 458-464. doi:10.1016/j.autrev.2006.03.011

[12] Swartz, R.D. (2009) Idiopathic retroperitoneal fibrosis: A review of the pathogenesis and approaches to treatment. American Journal of Kidney Diseases, 54, 546-553. doi:10.1053/j.ajkd.2009.04.019

[13] Swartz, R.D., Lake, A.M., Roberts, W.W., Faerber, G.J. and Wolf, J.S. (2008) Idiopathic retroperitoneal fibrosis: A role for mycophenolate motefil. Clinical Nephrology, 69, 260-268.

[14] Paz, Z. and Shoenfeld, Y. (2010) Antifibrosis: To reverse the Irreversible. Clinical Reviews in Allergy and Immunology, 38, 276-286. doi:10.1007/s12016-009-8157-7 